Publicación cuatrimestral. Vol. 5, No. 4 (Número Especial), Año 2021. Pág. 1-8 DATA MINING COMO SOPORTE PARA LA TOMA DE DECISIONES EN EL ENTORNO EMPRESARIAL

\title{
DATA MINING COMO SOPORTE PARA LA TOMA DE DECISIONES EN EL ENTORNO EMPRESARIAL
}

\author{
AUTORES: Jennifer Elizabeth Chóez Calle $\mathrm{1}^{*}$ \\ Victor Antonio Figueroa Castillo ${ }^{2}$ \\ Jennifer Xiomara Barreto Pin ${ }^{3}$ \\ Carlos Andrés Villacreses Parrales ${ }^{4}$ \\ Ing. Mario Javier Marcillo Merino ${ }^{5}$
}

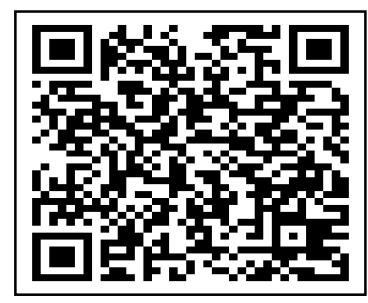

DIRECCIÓN PARA CORRESPONDENCIA: (jenniferelizachoezcalle@gmail.com)

Fecha de recepción: 26/03/2021

Fecha de aceptación: 30/05/2021

\section{RESUMEN}

La presente investigación da preámbulo sobre como el data mining sirve de soporte para la toma de decisiones dentro de un entorno empresarial, para ello se indaga en diversas fuentes sobre los procesos automatizados que se implementan en organizaciones, para tener un mayor apogeo en la industria, debido a que actualmente se generan millones de datos de información, por lo que se requiere aplicar la minería de datos, ya sean en diversos campos, empleando sistema de redes de conexión para la limpieza, recopilación y análisis de la información, que conlleve a las mejores tomas de decisiones, cabe recalcar que el data mining predice y detecta las tendencias, los patrones que se encuentra en el entorno del usuario como que es lo que mayormente compra, es decir sus gustos y necesidades, todo esto se obtiene mediante técnicas, algoritmos que se aplican para entender mejor los riesgos del mercado, y que estos diagnósticos sean mucho más precisos. El objetivo primordial de esta investigación científica es proporcionar información acerca de porque se aplica la data mining para la toma de mejores decisiones en los entornos empresariales, la metodología fue la revisión bibliográfica. El análisis de la investigación realizada demostró que actualmente en gran medida las organizaciones que aplican esta técnica.

PALABRAS CLAVE: información; mercado; patrones.

\footnotetext{
${ }^{1}$ Profesional en formación de la Carrera Tecnologías de la Información. Facultad de Ciencias Técnicas.Universidad Estatal del Sur de Manabí. Jipijapa, Manabí, Ecuador.https://orcid.org/0000-0001-6051-2479. Email:jenniferelizachoezcalle@gmail.com

${ }^{2}$ Profesional en formación de la Carrera Tecnologías de la Información. Facultad de Ciencias Técnicas.Universidad Estatal del Sur de Manabí. Jipijapa, Manabí, Ecuador.https://orcid.org/0000-0002-7810-9730. E-mail: victorfige@outlook.com

${ }^{3}$ Profesional en formación de la Carrera Tecnologías de la Información. Facultad de Ciencias Técnicas.Universidad Estatal del Sur de Manabí. Jipijapa, Manabí, Ecuador.https://orcid.org/0000-0002-9610-8002. E-mail: jenniferbarretopin@gmail.com

${ }^{4}$ Profesional en formación de la Carrera Tecnologías de la Información. Facultad de Ciencias Técnicas.Universidad Estatal del Sur de Manabí. Jipijapa, Manabí, Ecuador.https://orcid.org/0000-0002-4048-4316. E-mail: carlosvillacresesparrales23@gmail.com
}

(C) Universidad Estatal del Sur de Manabí. Jipijapa, Ecuador. 


\footnotetext{
${ }^{5}$ Ing. Mario Javier Marcillo Merino Master en Docencia Universitaria -Docente de Carrera Tecnologías de la Información en la Universidad Estatal del Sur de Manabí Jipijapa-Manabí-Ecuadormario_marcillo_merino@hotmail.com
}

\title{
DATA MINING AS SUPPORT FOR DECISION MAKING IN THE BUSINESS ENVIRONMENT
}

\begin{abstract}
This research gives a preamble on how data mining serves as support for decision-making within a business environment, for this, various sources are investigated about the automated processes that are implemented in organizations, to have a greater apogee in the industry, Due to the fact that currently there are millions of information data, therefore it is required to apply data mining, whether in various fields, using the connection network system for the cleaning, collection and analysis of information, leading to the best decision-making, it should be noted that data mining predicts and detects trends, the patterns found in the user's environment as what they mostly buy, that is, their tastes and needs, all this is obtained through techniques, algorithms that take risks to better understand market risks, and that these diagnoses are much more accurate. The primary objective of this scientific research is to provide information about why data mining is applied to make better decisions in business environments, the methodology was the literature review. The analysis of the research carried out showed that currently to a large extent the organizations that participated in this technique.
\end{abstract}

KEYWORDS: information; market; patterns.

\section{INTRODUCCIÓN}

Hoy en día la toma de decisiones dentro del entorno empresarial influyen de tal manera que con tan solo que una de ellas sea la errónea puede llevar al fracaso o deterioro de la misma, es por ello que las tecnologías cumplen un rol muy importante, debido a que mediante el uso de estas herramientas integran data mining para obtener una recopilación de datos de gran relevancia para la industrias u otras disciplinas, donde la competencia en ocasiones llevan gran ventaja es ahí donde intervienen las correctas estrategias a emplear después de conocer y evaluar los datos obtenidos del data mining.

En vista que los datos almacenados son un tesoro para las organizaciones, es en donde se guardan las interacciones pasadas con los clientes, la contabilidad de sus procesos internos, además representan la memoria de la organización. Tener memoria no es suficiente, hay que pasar a la acción inteligente sobre los datos para extraer la información que almacenan, este es el objetivo de la minería de datos.

Se aplican diversos patrones, técnicas, indicadores, aprendizaje automático, modelos que ayuden a interpretar el comportamiento que tienen cada uno de los clientes consumidores, sus tendencias, gustos, necesidades, entre otros, cabe recalcar que el data mining se enfoca en grandes bases de datos conocida como data warehouse o grandes almacenes de bases de datos donde a diario se almacenan gran cantidad de las compras que se realizan en línea, las visitas de las páginas web, trasferencias, depósitos bancarios y otros.

2 UNESUM-Ciencias. Publicación cuatrimestral. Vol. 5, Año 2021, No. 4 (Número Especial) 
Publicación cuatrimestral. Vol. 5, No. 4 (Número Especial), Año 2021. Pág. 1-8 DATA MINING COMO SOPORTE PARA LA TOMA DE DECISIONES EN EL ENTORNO EMPRESARIAL

La data mining es la clave principal a la hora de incluir una estrategia comercial, es decir no se puede avanzar en una idea o promoción sin antes conocer hacia dónde va a estar dirigía si esta idea va a ser acogida o no, es por ello que se aplica previamente y con mucho cuidado.

\section{DESARROLLO}

¿Qué es la minería de datos?

Se define a la minería de datos o data mining (DM) como el proceso de hallar outliers "anomalías", patrones y correlaciones en grandes conjuntos de datos para predecir resultados. Empleando una amplia variedad de técnicas, puede utilizar esta información para incrementar sus ingresos, recortar costos, mejorar sus relaciones con clientes, reducir riesgos y más.(SAS, 2018)

El uso de la minería de datos como soporte a decisiones en los negocios es más que aplicar redes neuronales o árboles de decisión sobre los datos por un lado está el descubrimiento del conocimiento en la base de datos y por otro lado están las técnicas estadísticas como el reconocimiento de patrones y algoritmos de aprendizaje entre otros.

El data mining posibilita, entre otros aspectos:

- Limpiar los datos de ruido y repeticiones.

- Extraer la información relevante y utilizarla para evaluar posibles resultados.

- Tomar mejores decisiones de negocio con mayor rapidez.

Enfoques en el mercado

En un mercado sobrecargado donde la competencia es cerrada, las respuestas se encuentran a menudo en los datos de sus consumidores. Las compañías de multimedia y telecomunicaciones pueden utilizar modelos analíticos para entender montañas de datos de clientes, ayudándoles así a predecir el comportamiento de sus clientes y ofrecer campañas altamente dirigidas y relevantes.(SAS, 2018)

Con conocimientos analíticos, las compañías de seguros pueden resolver problemas complejos concernientes a fraude, cumplimiento, gestión de riesgo y separación de clientes. Las compañías han utilizado técnicas de minería de datos para asignar precios a productos con mayor eficacia en líneas de negocios y hallar nuevas formas de ofrecer productos competitivos a su base de clientes existente.

Objetivo

Data mining dentro del entorno empresarial tiene como objetivo mejorar el acceso a la información está siendo cada vez más necesaria a la hora de tomar decisiones de empresa, se requieren procesos automáticos y reutilizables que ayuden a mejorar la competencia de los negocios obteniendo de forma rápida la información que es importante, descubriendo conocimiento y patrones en base de datos, para que esto pueda dar lugar, hace falta establecer medidas de evaluación del resultado derivado de la aplicación de las técnicas de minería de datos como analizar información que cambian en tiempo real.(Lopez, 2015)

Las empresas tiene que tener la capacidad de adaptarse a un cambio continuo, para aprender cómo resolver problemas y generar conocimiento para gestionar el flujo de información se dividen en dos, las aplicaciones que manejan las transacciones y las estadísticas que ayudan a convertir los datos en información útil para tomar decisiones a parte de las bases de datos donde se almacenan los datos importantes para evaluar y mejorar el funcionamiento de la cadena de

(C) Universidad Estatal del Sur de Manabí. Jipijapa, Ecuador. 
suministros y los análisis que facilitan la comprensión de las tendencias y patrones presentes en los datos.(Lopez, 2015)

La mayoría de los trabajos de minería de datos están orientados a la data warehouse, arquitectura de algoritmos, herramientas y técnicas utilizadas para agrupar los datos provenientes de múltiples bases de datos u otras fuentes de información en un repositorio común sobre el cual de harán consultas y análisis, se esta forma se consigue orientar los datos hacia el negocio.

La implementación de procesos de minería de datos a través de la aplicación de técnicas estadísticas avanzadas y nuevos métodos de extracción de conocimiento en grandes bases de datos se pueden determinar las características contables de empresas más rentables al igual que el perfil de sus clientes, es necesario, por tanto, un análisis exploratorio profundo de la base de datos y el empleo de métodos que hagan que dichos modelos sean menos sensibles a los casos estadísticos.

¿Cómo implementar el DM en una organización?

Se debe analizar los factores, determinar los procesos, predecir o estimar las variables, de los comportamientos que se vayan a obtener de la recopilación de información para ello estos deben ser secuenciales aplicando el DM en base a los lenguajes de la inteligencia artificial, redes neuronales, sistemas expertos, programación genética y árboles de decisión, todas estas técnicas se involucran para establecer un sistema de conexión para la evaluación y presentación de la información recabada y posterior tomar decisiones aplicando estrategias que sobresalgan en el entorno empresarial.

¿Cómo el DM favorece a una organización?

Las áreas en las que se aplica el DM son banca, finanzas, seguros, marketing, políticas sanitarias o demográficas, todos los datos corresponde al éxito de las operaciones empresariales, utilizadas para detectar fraudes o riesgos dentro de los sistemas críticos y tiene todas las características que pueden ser de interés para un analista, el cual puede analizar estas anomalías para descubrir lo que realmente está pasando.

De tal manera que ayuda a las organizaciones a encontrar la información más importante y relevante. Esta información se puede utilizar para crear modelos que pueden ayudar a hacer predicciones de cómo las personas o sistemas se computarán para que se puedan anticipar a él. (Power Data, 2017)

Etapas

La minería de datos es la mejor forma de obtener los insights, con base, obviamente en los mejores datos. Necesitas trabajar con una estrategia conjunta de captación de datos de calidad y la utilización de un método robusto para analizar los datos, con el fin de obtener los insights más valiosos a partir de éste. Para esto se aplican tres etapas.

- La exploración

- La construcción de un modelo.

- La validación

El objetivo de estas tres etapas es analizar los datos y encontrar oportunidades, problemas, al igual que soluciones con base en el comportamiento de los negocios, además de la información obtenida con los datos. El análisis sucede por cuenta de algunos algoritmos matemáticos avanzados que categorizan la información y hacen análisis predictivos presentando respuestas los llamados insights- para problemas de negocios. La construcción de modelos patrones entra exactamente aquí, o sea, el Data Mining los crea para su análisis y encuentra resultados similares.

4 UNESUM-Ciencias. Publicación cuatrimestral. Vol. 5, Año 2021, No. 4 (Número Especial) 
Publicación cuatrimestral. Vol. 5, No. 4 (Número Especial), Año 2021. Pág. 1-8 DATA MINING COMO SOPORTE PARA LA TOMA DE DECISIONES EN EL ENTORNO EMPRESARIAL

Con eso, descubre de forma automatizada información relevante para la toma de decisiones de las empresas. (Mexico Blog, 2018)

Técnicas

Existen diferentes técnicas que en las organizaciones emplean para la toma de decisiones estas se basan en todos los millones de datos que poseen, entre ellas se destacan las siguientes:

- Razonamiento estadístico, se utilizan para obtener datos del pasado.

- Visualización, se usan para generar un feeling de calidad con los datos.

- Procesamiento paralelo, para agilizar el procesamiento de consultas.

- Apoyo a la toma de decisiones, basados en la teoría de la decisión.

- Aprendizaje automático, consiste en aprender reglas a partir de los datos, aprender experiencias del pasado con respecto a alguna medida de rendimiento. (Lopez, 2015)

Todas estas técnicas las aplican usando una clasificación, es decir asignando una categoría a cada dato obtenido, basándose en un clustering, en pocas palabras se buscan características comunes entre millones de datos, se analizan toda la información recabada, posterior a ello se aplica un sistema de interconexión de datos finalmente se construyen lógicas para organizar y mostrar los datos y así lleguen a una resolución para ver qué estrategia comercial aplicar e innovar en el entorno.

Las estrategias que se deben aplicar conforme a todos los datos que se han obtenido de la data mining deben adaptarse a un mercado globalizado y esto incide de forma directa en su sistema estructural.

La creación de un modelo de clústeres es el primer paso porque, aunque el algoritmo siempre generará los clústeres adecuados, el usuario debe comprender de qué manera difiere cada clúster de los demás. El análisis de clúster no genera ninguna conclusión en sí mismo, y habrá que analizar los resultados para buscar esas relaciones ocultas entre los atributos que nos permitan determinan la segmentación, en este caso, de los clientes. (Espinosa , 2010)

Se emplean herramientas de soporte que sirvan para expandir las capacidades para procesar grandes volúmenes de información durante el proceso de toma de decisiones en el entorno empresarial.

¿Por qué usar Data Mining en tu organización?

Ya seas una PyME o una empresa de gran porte, tus equipos poseen una tonelada de datos a disposición: facturas, registros de inventarios, plantillas y más plantillas. Con seguridad, puedes usar ese montón de datos el Big Data a tu favor.

Ventajas

- Es la anticipación de tendencias de tu mercado. Esta ayuda a las organizaciones a prever tendencias como, por ejemplo, en cuáles épocas del año la interacción de los consumidores es más elevada e intensa, pudiendo así anticipar momentos semejantes a futuro.

- La posibilidad de evitar y contrarrestar crisis corporativas. Con el monitoreo de los datos en tiempo real de los medios sociales, es posible validar cómo los clientes observan a la compañía.

- La herramienta de análisis de información consigue ofrecer insights importantes para la toma de decisiones y para la creación de acciones que fortalezcan a la marca. (Mexico Blog, 2018)

(C) Universidad Estatal del Sur de Manabí. Jipijapa, Ecuador. 
Indicadores en la toma de decisiones aplicando data mining

Para esto se integran el análisis de clúster el cual ofrece un método para agrupar valores de datos basado en similitudes dentro de estos, esta técnica segmenta distintos elementos en grupos según el grado de asociación entre los elementos. El grado de asociación entre dos objetos es máximo si pertenecen al mismo grupo y mínimo si no pertenecen al mismo grupo. Se forma un número determinado o especificado de grupos, o clústeres, lo que permite clasificar matemáticamente cada valor de los datos en el grupo adecuado.

- Indicador de la bondad aportan la idea acerca del error que se comete al aplicar con modelo determinado, este indicador nos da a conocer la fortaleza estadística del resultado.

- Indicador de relevancia del resultado, da a conocer la importancia del resultado y la aportación a la situación actual, la frecuencia de utilidad del resultado.

- Indicador de novedad del resultado, determina la importancia de una regla, ante una serie de reglas ya establecidas para un área de conocimiento determinada.

- Indicador de aplicabilidad del resultado, se basa en la simplificación de los modelos y la forma de representar los datos obtenidos, para transformar el conocimiento obtenido y aplicarlo al negocio.

\section{MATERIALES Y MÉTODOS}

En la presente investigación que se plantea se aplicaron diferentes materiales que permitieron dar continuidad tanto al desarrollo como fuentes bibliográficas las cuales permitieron sustentar la indagación, así mismo como artículos de revistas, científicos; entre otros.

Para abordar el desarrollo de la investigación se emplearon y utilizaron métodos científicos tales como: métodos teóricos en el cual destaca el histórico-lógico los mismos que permitieron determinar los antecedentes presentes en la investigación sobre la data mining como soporte para la toma de decisiones en el entorno empresarial denotando sus ventajas, indicadores para ser aplicada en las industrias, el método análisis-síntesis para analizar y sintetizar las revisiones bibliográficas de diferentes artículos, revistas, periódicos, libros, sitios web; por otro lado el método referencial-bibliográfico que contribuyo para tener un fundamento sustentable, precisa y correcta.

\section{RESULTADOS}

La utilización de la data mining en los diferentes ámbitos desde la educación hasta el ámbito empresarial, en la Figura $N^{0}$ 1se muestra el esquema de la arquitectura de una data warehouse, donde los tres niveles tienden a tener la referencia de un almacén de datos como la recolección, el almacenamiento y entrega de los datos, los cuales se obtienen utilizando los siguientes métodos de extracción como los clustering, algoritmos, herramientas y técnicas. 
Figura $\mathbf{N}^{\mathbf{0}} \mathbf{1}$ : Esquema de la arquitectura de referencia de un data warehouse Fuente: Digital Guide IONOS, datos obtenidos de la Web

Las áreas de aplicación de la minería de datos se abordan en diferentes campos como los son la agricultura, Biología, educación, medicina, informática, ergonomía, entretenimiento, eléctrico, entre otros; es por ello que el la Figura $N^{o}$ 2se detalla en porcentaje de la obtención de los datos que se generan día tras día, logrando almacenar, clasificar, analizar y compartir con otras entidades para conseguir resultados en beneficio de la organización.

$$
\text { Áreas de aplicación }
$$

$$
\begin{aligned}
& \text { = Agricultura }=\text { Biología }=\text { Educación } \mid \text { Eléctrico } \\
& \text { = Entretenimiento }=\text { Ergonomía }=\text { Informática } \| \text { Medicina }
\end{aligned}
$$

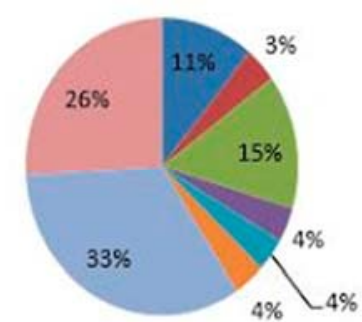

Figura $\mathbf{N}^{0} 2$ : Áreas de aplicación aplicando la minería de datos Fuente: WEKA, datos obtenidos de la Web

(c) Universidad Estatal del Sur de Manabí. Jipijapa, Ecuador. 


\section{DISCUSIÓN}

El data mining, en la actualidad ha tenido una creciente demanda en muchos ámbitos en más denotado es en cuanto a los entornos empresariales en la inclusión de los negocios debido a la enorme cantidad de datos que demandan y emiten las empresas por conocer más allá de los datos como las tendencias de mercado, los gustos, la competencia, éstos se convierte en una herramienta estratégica siempre y cuando sea aplicada correctamente, proporcionando elevar los niveles de competencia en el mundo de los negocios.

Las tomas de decisiones se vuelven efectivas, dependiendo de la rapidez con que se identifiquen y analicen la información de gran relevancia, para aplicar la minería en los entornos empresariales, empleando los patrones de búsqueda teniendo un impacto significativo en los entornos empresariales, para incrementar las ventas y adquisición de clientes en las empresas.

\section{CONCLUSIONES}

Se colige que la integración de la data mining en el entorno empresarial es de gran ayuda debido a que no solo se conocer y obtener patrones, comportamiento es decir información relevante, sino que a la vez se aplican estrategias que posterior a utilizarlas podría tener un gran crecimiento la organización, aplicando redes, patrones, inteligencia artificial y así recopilar los datos de grandes bases de datos, como los gustos o tendencias de los clientes y el entorno en el que se encuentran. Las técnicas como lo son las redes neuronales, la clasificación de la información, los clústeres de los datos que se tienden a ser comunes como las características de los clientes, productos y así emplear las herramientas como lo son la verificación y descubrimiento, es decir predecir y detectar lo que puede afectar o mejorar en las empresas.

\section{REFERENCIAS BIBLIOGRÁFICAS}

Espinosa , R. (05 de marzo de 2010). Archive for the 'Data Mining' Category. Obtenido de churriwifi.wordpress.com: https:/churriwifi.wordpress.com/category/data-mining/

Lopez, R. (13 de mayo de 2015). Minería de datos como soporte a la toma de decisiones. Obtenido de comunidad.iebschool.com: https://comunidad.iebschool.com/bigdata/2015/05/13/mineria-de-datos-comosoporte-a-la-toma-de-decisiones/

Mexico Blog. (18 de junio de 2018). DATA MINING: ¿CÓMO USARLA EN TU EMPRESA? Obtenido de blogmexico.comstor.com: https://blogmexico.comstor.com/data-mining-como-usarla-en-tu-empresa

Power Data. (22 de febrero de 2017). ¿Qué es el data mining y cómo puede ayudar a crear valor empresarial? Obtenido de blog.powerdata.es: https://blog.powerdata.es/el-valor-de-la-gestion-de-datos/que-es-el-datamining-y-como-puede-ayudar-a-crear-valor-empresarial

SAS. (2018). Minería de datos. Obtenido de sas.com: https://www.sas.com/es_mx/insights/analytics/datamining.html 